\title{
$\nabla$
}

\section{Confluent and reticulate papillomatosis of Gougerot- Carteaud and obesity: dermoscopic findings}

\author{
Fred Bernardes Filho ${ }^{1}$ \\ Bernard Kawa Kac ${ }^{1}$
}

\author{
Maria Victória Quaresma ${ }^{1}$ \\ José Augusto da Costa Nery ${ }^{1}$
}

\author{
Fernanda Coelho Rezende ${ }^{1,2}$ \\ Luna Azulay-Abulafia ${ }^{1,3}$
}

Abstract: Confluent and reticulated papillomatosis of Gougerot and Carteaud is a dermatosis that despite showing characteristic clinical signs is often poorly recognized and diagnosed. The authors present a case with extensive skin involvement, discuss its association with obesity and describe dermoscopic findings making the histopathological correlation.

Keywords: Hyperpigmentation; Obesity; Papilloma

\section{INTRODUCTION}

Confluent and reticulated papillomatosis (CRP) of Gougerot-Carteaud is characterized by the presence of brown, spotted, warty papules measuring about $5 \mathrm{~mm}$ in diameter that coalesce in the center and have a peripheral reticulate pattern, usually forming plaques often desquamative and with imprecise limits. ${ }^{1,2,3}$ It is more frequent among the female sex, nonwhite race, and overweighted individuals, occurring most usually around the second or third decades of life. The lesions are located in the interscapular, epigastric and intermammary regions, sparing, in general, the mucosae and palmoplantar areas. ${ }^{1,3}$ In extensive cases it may affect the neck, shoulders, axillae and pubic areas. ${ }^{1}$

The differential diagnosis is made mostly with acanthosis nigricans and pityriasis versicolor. ${ }^{1,2,3}$ However, because of their similar and overlapping clinical features, some authors consider PCR and acanthosis nigricans as one spectrum of the same entity. ${ }^{2}$

Dermoscopy is a noninvasive and quick auxiliary method that allows the correlation of structures, which are not visible to the naked eye, with histopathological examination of the lesions, thus being an increasingly common tool in clinical practice. ${ }^{4}$

Hence, we present an extensive case of PCR that evolved with significant reduction of lesions after the patient adopted measures to control his weight, including physical activity and diet, and we also describe dermoscopic findings of lesions and their histopathological correlation.

\section{CASE REPORT}

Thirty-six year-old male patient reported the presence of desquamative and pruritic hypertrophic lesions on the trunk and back for the last 14 years. He related several treatments with topical medications, without satisfactory results. The patient denied comorbidities and was not using any medication at that moment. Dermatological examination showed macules and papules with a velvety and scaly surface, converging centrally and with a peripheral reticulate pattern (Figure 1). The patient presented axillary, cervical and pubic hyperpigmentation. Weight: $98 \mathrm{~kg}$. Height: 1.78 m. BMI: 30.9 (obesity class I). Dermoscopy evidenced a brownish pigmentation of poorly defined borders, covered with white scales and a pattern of "sulci and gyri" (Figure 2). Direct mycological examination of the lesion was negative and culture did not show fungi growth. Lesion biopsy on the dorsal area demonstrated mild parakeratosis, compact hyperkeratosis, hyperpigmentation of the basal layer, papillomatosis and superficial perivascular mononuclear infiltrate (Figures 3 and 4). PAS staining was negative. Diagnosis of confluent

\footnotetext{
Received on 20.04.2013.

Approved by the Advisory Board and accepted for publication on 26.06.2013

* Work performed at Professor Rubem David Azulay Dermatology Institute - Hospital Santa Casa da Misericórdia do Rio de Janeiro (IDPRDA - SCMRJ) - Rio de Janeiro (RJ), Brazil.

Conflict of interest: None

Financial funding: None

Santa Casa da Misericórdia do Rio de Janeiro (SCMRJ) - Rio de Janeiro (RJ), Brazil.

Gama Filho University (UGF) - Rio de Janeiro (RJ), Brazil.

Rio de Janeiro State University (UERJ) - Rio de Janeiro (RJ), Brazil. 


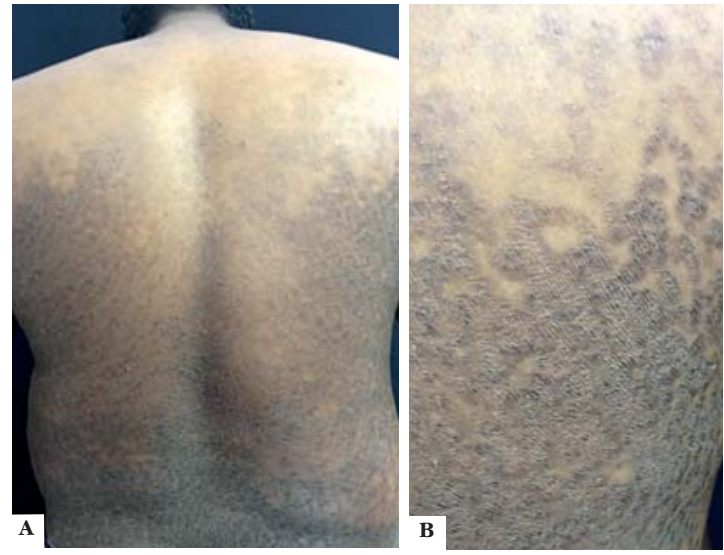

FIGURE 1: (A-B) Macules and papules of velvety and desquamative surface, centrally confluent and with a peripheral reticulate pattern

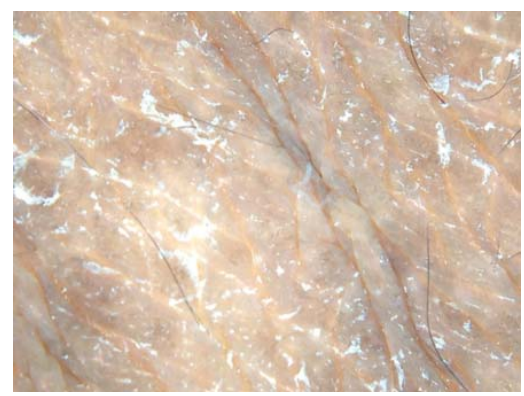

Figure 2:

Brownish coloration with poorlydefined borders, covered in white scales and "sulci and gyri" pattern

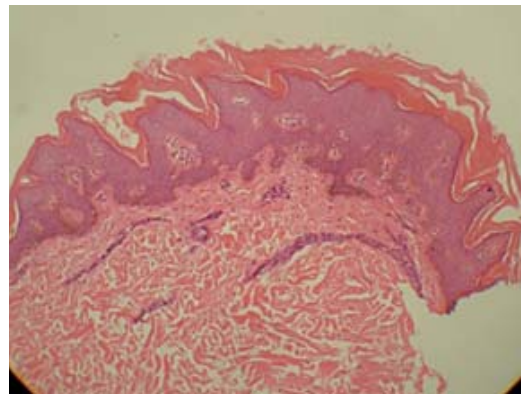

Figure 3:

Discreet parakeratosis, compact hyperkeratosis, basal layer hyperpigmentation, papillomatosis and superficial perivascular mononuclear infiltrate

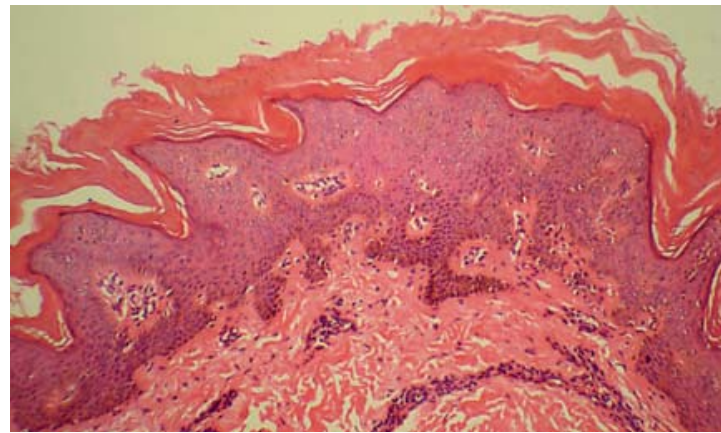

FIGURE 4: Higher magnification showing in detail parakeratosis, compact hyperkeratosis, papillomatosis and pronounced irregular hyperpigmentation of the basal layer and reticulated papillomatosis of Gougerot and Carteaud was established and weight loss was initially suggested. The patient returned after 45 days presenting a significant reduction in the number of lesions (Figure 5).

\section{DISCUSSION}

PCR is a rare disease, often underdiagnosed by dermatologists. Its cause is unknown. ${ }^{2,3}$ Associations with endocrine disorders, such as obesity, type 2 diabetes mellitus, hirsutism, Cushing's syndrome, menstrual abnormalities, and thyroid dysfunction have been suggested, however endocrinopathy is not a sine qua non condition for the development of the disease. Some authors also advocate connections with vitamin A deficiency, genetic factors, photosensitivity, cutaneous amyloidosis, tissue reaction to skin colonization by lipophilic yeasts of genus Malassezia, staphylococci or Propionibacterium acnes, and especially keratinization defects. ${ }^{1-3}$

Body mass index (BMI) is a rapid and easy method for evaluating the optimal weight of individuals and also an international predictor of obesity adopted by the World Health Organization (WHO) (Table 1). ${ }^{5}$ Obesity is recognized as a multifactorial disease that predisposes to several other comorbidities, particularly cardiovascular illnesses. ${ }^{6}$ In dermatology,

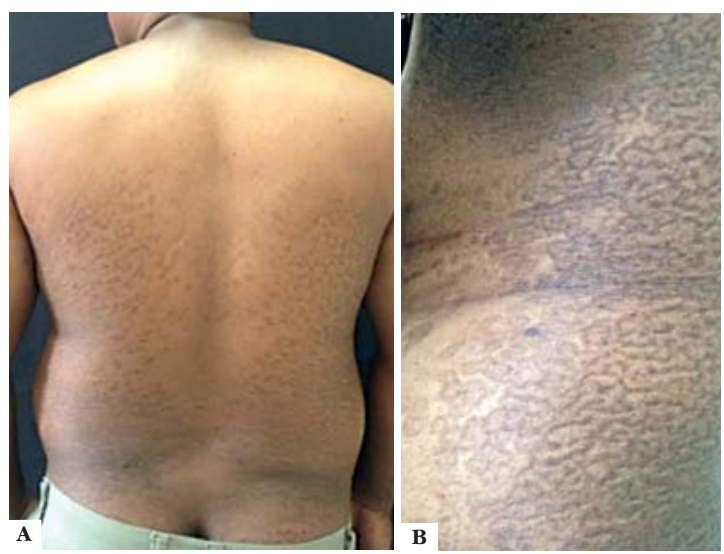

FIGURE 5: Significant reduction in the number of lesions 45 days after adopting weight loss measures

Table 1: Obesity classification according to body mass index (BMI)

\begin{tabular}{ll}
\hline BMI & Classification \\
\hline$<18.5$ & Underweight \\
$18.6-24.9$ & Healthy \\
$25-29.9$ & Overweight \\
$30.0-34.9$ & Obesity Class I \\
$35.0-39.9$ & Obesity Class II (severe) \\
$\geq 40.0$ & Obesity Class III (morbid)
\end{tabular}


Chart 1: Medications used in topic and systemic treatment of CRP

\begin{tabular}{|ll}
\hline Topical use & Oral use \\
\hline Corticosteroids, ketoconazole, 20\% sodium thiosulfate, sele- & Retinoids: isotretinoin, etretinate, acitretin, tazarotene, \\
nium sulfide, salicylic acid, hydroquinone, tacrolimus, anti- & $\begin{array}{l}\text { antifungals and antibiotics: doxycycline, minocycline and } \\
\text { fungals, 5-fluorouracil, phototherapy, progesterone, coal tar, } \\
\text { erea, liquid nitrogen and dermabrasion. }\end{array}$
\end{tabular}

it is markedly associated to acanthosis nigricans, psoriasis, and hidradenitis suppurativa, among others. ${ }^{6-9}$ We emphasize its connection to CRP and highlight the improvement of this dermatosis with weight control measures.

After a dermoscopic-histopathological correlation, the authors propose that the appearance of brownish pigmentation with poorly defined edges, covered with white scales would correspond to basal layer hyperpigmentation, parakeratosis, compact hyperkeratosis, and that the aspect of "sulci and gyri" (depressions and elevations) would correspond to papillomatosis.

PCR treatment is often frustrating, because there is the possibility that it may not respond proper- ly, and also the recurrence of symptoms that may occur after therapy discontinuation. Usually therapeutic success is not achieved with monotherapy and the rates of dissatisfaction are very expressive. . $2,3,10^{2}$ Several drugs used in the treatment of PCR are described in chart 1 and they present varying response rates.

Because our case depicts an exuberant clinical form of confluent and reticulated papillomatosis of Gougerot-Carteaud, the clinical improvement of lesions after weight loss and also for demonstrating the standard "sulci and gyri" at dermoscopy, it constitutes a relevant publication.

\section{REFERENCES}

1. Ferreira LM, Diniz LM, Ferreira CJM. Confluent and reticulated papillomatosis of Gougerot and Carteaud: report of three cases. An Bras Dermatol. 2009;84:78-81.

2. Cockerell CJ, Larsen F. Confluent and Reticulated Papillomatosis. In: Bolognia JL, Jorizzo JL, Rapini RP, editors. Dermatology. 2th. New York: Mosby Elsevier; 2008. p. 1677-8.

3. Davis MD, Weenig RH, Camilleri MJ. Confluent and reticulate papillomatosis (Gougerot-Carteaud syndrome): a minocycline-responsive dermatosis without evidence for yeast in pathogenesis. A study of 39 patients and a proposal of diagnostic criteria. Br J Dermatol. 2006;154:287-93.

4. Rezze GG, Soares de Sá BC, Neves Rl. Dermoscopy: the pattern analysis. An Bras Dermatol. 2006;81:261-8

5. Halpern A, Mancini MC. Obesidade (Obesity). In: Lopes AC. Tratado de Clínica Médica. 2. ed. São Paulo: Roca; 2006. p. 3555-67

6. Tamega AA, Aranha AMP, Guiotoku MM, Miot LDB, Miot HA. Association between skin tags and insulin resistance. An Bras Dermatol. 2010;85:25-31.

7. Duarte GV, Follador I, Cavalheiro CM, Silva TS, Oliveira Mde F. Psoriasis and obesity: literature review and recommendations for management. An Bras Dermatol. 2010;85:355-60.

8. Schmitt JV, Bombonatto G, Martin M, Miot HA. Risk factors for hidradenitis suppurativa: a pilot study. An Bras Dermatol. 2012;87:936-8.

9. Schmitt JV, Bombonatto G, Martin M, Miot HA. Acanthosis nigricans in obese women in a mixed-race population: a marker of metabolic disturbances. An Bras Dermatol. 2002;77:537-43.

10. Bowman PH, Davis LS. Confluent and reticulated papillomatosis: Response to tazarotene. J Am Acad Dermatol. 2003;48:S80-1.

\author{
MAILING ADDRESS: \\ Fred Bernardes Filho \\ Rua Marquês de Caxias, 9 Sobrado - Centro \\ 24030-050 - Niterói - RJ \\ Brazil \\ E-mail:f9filho@gmail.com
}

How to cite this article: Bernardes Filho F, Quaresma MV, Rezende FC, Kac BK, Nery JAC, Azulay-Abulafia L. Confluent and reticulate papillomatosis of Gougerot-Carteaud and obesity: dermoscopic findings. An Bras Dermatol. 2014;89(3):507-9. 